\title{
Microgravity: A Tool for Protein Drug Development
}

\author{
*JohnPaul O. Enemali, Funmilola A. Oluwafemi, Solomon J. Hussaini, Ishaya B. Daniel, Omede Ameh, Abubakar Adamu, \\ Olufemi A. Agboola
}

Engineering and Space Systems (ESS) Department, National Space Research and Development Agency (NASRDA), Km 17 Airport Road, P.M.B. 437, Abuja, Nigeria.

*Corresponding author's E-mail: enemalij@yahoo.com

Received: 14-06-2020; Revised: 21-08-2020; Accepted: 28-09-2020; Published on: 20-10-2020.

\begin{abstract}
For the past two decades diverse technological advancement in X-ray crystallography have resulted in faster determination of structures of large macromolecules especially proteins. Although the capacity to produce diffraction-quality crystals suitable for exhaustive structural analysis remains a bottleneck, even with the combined automated technology cum site directed mutagenesis. Research in the crystallization of protein in microgravity promises to proffer solution to some of the grey areas as crystals of better quality are produced in the microgravity compared to the Earth's environment. This is used extensively in drug development especially monoclonal antibodies (MABs); and in other applications has been acknowledged by some group of scientists and those of the International Space Station (ISS). This paper recapitulates the account of some of the work done by these scientists on protein crystals yielded in microgravity and the application of the crystals in MABs drug development and other important areas.
\end{abstract}

Keywords: Microgravity, Proteins, Crystallization, Drug development, Monoclonal antibodies (MABs).

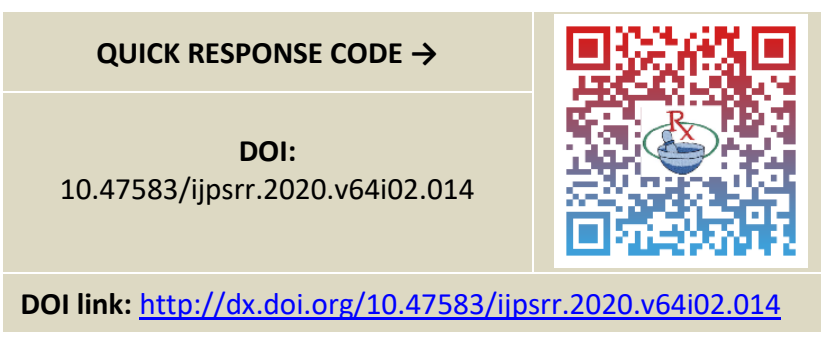

\section{INTRODUCTION}

$\mathrm{P}$

roteins are very important biological macromolecules that play a key role in the complex mechanism of life. They interact with other biological molecules in specialized ways because they possess active sites. Their mode of interaction determines their biological function. Apart from their usual intervention in biological systems regulation, reproduction and maintenance mechanisms of living organisms, they act as catalysts and carryout other biological functions. Contrarily, they are also the cause of some diseases and disorders ${ }^{1}$. This knowledge has been employed by pharmaceutical companies to develop novel drugs that target only affected macromolecule and inhibit the active site of macromolecules with the knowledge of their threedimensional (3D) structure ${ }^{2}$. With detailed knowledge of the 3D structure of biological macromolecules biochemists and biological scientists are able to restrict the choice of drugs to be tested, this also enable scientist to synthesize drug like monoclonal antibodies (MABs) that target solely a particular macromolecule using a lucid drug method strategy. This implies that a drug is able to bind effectively to the macromolecule inhibiting its biological role without having any effect on other macromolecules ${ }^{1}$.
Often the immune system is combated by some diseasecausing substances resulting in ailments. Pharmaceutical companies in a bid to combat disorders have developed countless techniques to enhance the immune system integrity ${ }^{3}$. A novel strategy is the development of drugs known as monoclonal antibodies that target specific attackers, igniting the immune cells into action.

MABs are used extensively in basic biomedical research, in diagnosis of disease, and in treatment of illnesses, such as infections and cancer. MABs are also concocted proteins that bind specifically to any (almost) given substances including those cancer-causing agents ${ }^{4}$.

For MABs to be effective it must be administered to patients in large quantities thereby making it challenging for medical practitioners and recipients. This is because the mode of administration of MABs therapy must be through injections or intravenous infusions. Apart from the task of doctors preparing injections; it is also painful for the patients who receive these injections, unlike the conventional pills that are easily swallowed. It therefore means that if MABs must be more effective it should be designed in form of pills. Development of these pills may therefore require crystallization of the proteins ${ }^{3}$.

Before now, X-ray crystallography remained the best method of crystallizing biological molecules, yet determining the crystal structure of all the proteins from an organism is onerous ${ }^{5}$. In spite the improvement made due to the combination of automated crystallization systems cum protein engineering (site-directed mutagenesis) that has enhanced crystallization attainment, there persists many other proteins that either cannot be crystallized or even when crystallized are 
insufficient qualities that can support X-ray structure determination. In an attempt to address this problem, international groups of scientists has explored the use of the microgravity environment to crystallize molecules. Today, space research is changing the trend ${ }^{6}$ as there are proofs that advanced research on the International Space Station is studying the structure of proteins by crystallizing MABs in microgravity. Investigation ab binitio shows that protein crystals developed in microgravity can produce substantively superior structural information than can be acquired from crystals yielded on Earth. These findings have been hinged on some factors such as the absence of sedimentation which prevents protein crystals from plummeting to the bottom of the containers on which they are grown as they do on their counterpart ground condition ${ }^{7}$. In the same vein they are not as likely to be affected by other crystals growing in the solution. Also, convective flows are also greatly reduced in microgravity and so crystals grown in a much more inert environment yield better quality. Therefore, space is an excellent environment to study complex, three-dimensional proteins. Through this effort, more concentrated and highquality mixtures that can be administered to patients more efficiently are developed as drugs ${ }^{3,5}$.

By crystallization, detailed knowledge on how biological molecules interact to form complex supramolecular assemblages is gotten as well as information on the structure that provides a great understanding on the fundamental mechanisms on the structure and function of biomolecules is determined ${ }^{7}$. Furthermore, knowledge of binding sites at atomic details through crystallography permits a lucid drug design, necessary for probing of novel medicines $^{8}$. This discovery has made protein crystallography useful at all level spanning from drug development to target identification and selection ${ }^{9,10}$. This review examines the performance of proteins in microgravity in comparison to the ground counterpart in drug development.

\section{MICROGRAVITY}

The National Aeronautics and Space Administration (NASA) stated that a microgravity environment is one in which the apparent weight of an object is little compared to its real weight due to gravity. It is the presence of minute gravity ${ }^{11}$. Furthermore, there is a feeling of weightlessness. The exceptional environment of microgravity experienced in space indicates that there is a prospect for discovery and development of novel drug that cannot be replicated on Earth. This is often shown from the generation of higher protein crystals to the identifiable evidence and validation of new drug targets to microarray examination of transcripts constricted by microgravity ${ }^{10}$. Hence, microgravity environment has been identified as a more proficient environment for superior protein crystal yield for drug development.

\section{PROTEIN STRUCTURE AND FUNCTION}

Proteins are large biomolecules or macromolecules, comprising of one or more long chains of residues of amino acid. Proteins are the most versatile macromolecules in living systems and serve crucial functions in essentially all biological processes and systems. They act as catalysts, carry and store other molecules like oxygen, provide mechanical support and immune defence, produce movement, carry nerve impulses and regulate growth and differentiation. Proteins have four levels of structures, which are primary, secondary, tertiary and quaternary ${ }^{12}$. The structure of protein is relevant to its function in the biological system.

\section{Primary structures of protein}

The primary structure of protein is a linear polymer with a series of amino acids. These amino acids are connected by Carbon-Nitrogen ( $\mathrm{C}-\mathrm{N}$ ) bonds, also known as peptide bonds. The formation of the peptide bonds produces water molecules as a by-product when an amino acid N-terminal loses hydrogen and another amino acid $C$ terminal loses hydroxyl group. Amino acid in a polypeptide chain is a unit, generally known as a residue. Such chains have a planar backbone, since the peptide bonds have double bond characteristics due to the resonance between carbonyl carbon and nitrogen in which the peptide bonds are formed $^{13}$. The primary structure of each protein is absolutely known by the specific genes.

\section{Secondary structures of protein}

The secondary structure stems from the hydrogen bonds that formed between polypeptide back bone atoms. The hydrogen bonds will form between the partially negative atom for oxygen and the partially positive atom for nitrogen. Many proteins have parts of their polypeptide chains that are either coiled or folded into patterns leading to the protein's form. Many of these spindles and folds occur so often that names are given. The alpha-helix and the beta-pleated layer are two folds that are extremely common in biochemistry. The alpha-helix is a right-hand helical coil that is held together between each fourth amino acid by hydrogen bonding. The other secondary structure is the sheet with beta-pleat. Two distinct regions of a polypeptide chain lay side in this structure and are bound by hydrogen bonds ${ }^{14}$.

\section{Tertiary structures of protein}

The tertiary structure is the structure that makes polypeptide chains functional. That protein has a particular three-dimensional shape at this point and features functional groups on its outer surface that allows it to interact with other molecules and give its unique function ${ }^{15}$.The arrangement is done with the help of chaperones, which move around the protein chain, bringing together different groups in the chain to help them form bonds. Such interacting amino acids are usually far away on the chain. The main interactions that make-up the tertiary structures of protein are hydrophobic 
interactions, disulphide bridges, ionic bonds and hydrogen bonds. These direct the twisting and bending which helps the protein molecule achieve a stable state ${ }^{16}$.

\section{Quaternary structures of protein}

A protein's quaternary structures are the aggregation of several protein chains or subunits into tightly packed arrangement. The subunits each have their own central, secondary and tertiary structure. The subunits are held in between non-polar side chains by hydrogen bonds and van der Waal forces ${ }^{17}$. The subunits in a quaternary structure have to be specifically arranged for proper functioning of the whole protein. Any change in the structure of the subunits or how they are related causes notable changes in biological activity. Example of a quaternary structure of a protein is haemoglobin ${ }^{18}$.

\section{PROTEIN CRYSTALLIZATION}

Crystallisation of proteins is the process of forming a protein crystal. Among other uses, protein crystals are useful for the study of protein structures for use in medicine. Proteins are dissolved in an aqueous atmosphere and sample solution during the process of protein crystallization until they enter the supersaturated state $^{19}$. This supersaturated state allows researchers to study the internal structure of proteins. Different methods are used to reach that state such as vapour diffusion, micro batch, micro dialysis, and free-interface diffusion. It is difficult to develop protein crystals, as the process is affected by many factors, including $\mathrm{pH}$, temperature, ionic activity in the crystallization solution and gravity ${ }^{19}$. Once properly developed, these crystals can be used in structural biology to study the molecular structure of the protein, particularly for various industrial or biotechnological purposes, such as developing cancer treatment, drugs and other therapeutic options ${ }^{20}$.

\section{BENEFITS OF PROTEIN CRYSTALLIZATION IN MICROGRAVITY}

Crystallization of organic molecules can lead to improvements in drug-development, formulation, manufacturing, and storage ${ }^{21}$. Protein crystallization in microgravity is done to improve the way crystals are grown on Earth. The information gained from experiments performed on protein crystallization in space could speedup the process for drug-development, benefiting humans around the world, while these crystals themselves also could be used in research on certain diseases around the world $^{22}$. Understanding of how different proteins crystallize in microgravity gives researchers a deeper-view into how these proteins function ${ }^{23,24}$.

A pure crystal can contain a single protein of thousands of copies. Once such crystals are returned to the Earth and exposed to an X-ray beam, the pattern of the X-ray diffraction can be used to mathematically map a protein's structure ${ }^{23}$.

\section{Development of new biological based drugs}

Crystal growth is important for the development of new, biologically based drugs, providing tougher weapons for physicians fighting human diseases. Pharmaceutical companies have developed different ways to fight ailments such as arthritis, cancer etc. but the best way to fight them has not been got. A new generation of drugs targets specific attackers, sparking immune cells into action. These drugs, called monoclonal antibodies (MABs), are engineered proteins that bind themselves to substances that cause disease. MABs can be fashioned for almost any target inside a cell or on cell's surface, allowing fewer side effects and better specificity than conventional therapies. They include top-selling drugs used to treat several types of inflammation and cancer ${ }^{25}$.

Since MABs have to be dispensed in large quantities intravenously for them to be effective, this makes it difficult to administer to patients. Now, research on the ISS is changing that by taking MABs into space and crystallizing them in microgravity. Several researches are on-going working toward high concentration, high quality mixtures that can be given to patients more efficiently ${ }^{25}$.

With large crystals gotten from microgravity environment, scientists on the ground can use X-ray crystallography to determine how the protein is organized. Determining protein structures helps researchers design new drugs ${ }^{26}$.

These high concentrations of protein crystals could improve the way patients receive MABs to treat a wide range of diseases, because patients to receive MABs usually have to wait for several hours to receive the full dose. In microgravity, the highly concentrated suspensions of crystallized proteins produced, could be given in a simple shot. The fluid would look similar to milk, opaque with high concentrations of crystallized MABs that makes it appear white.

Highly concentrated $\mathrm{MAB}$ mixtures will also be more efficient to ship and store. Currently, different components of MAB treatments are manufactured at different sites and afterwards shipped overseas or to different places to be formulated into drugs 25 .

\section{New ways to prevent and treat cancer}

Frederick National Laboratory for Cancer Research aim to use the ISS National Lab to crystallize KRAS proteins. KRAS is the most frequently mutated member of the RAS family of genes. The mutations that account for over $30 \%$ of all human cancers. Mutations in the KRAS gene are accountable for $95 \%$ of pancreatic ductal adenocarcinoma (the most common type of pancreatic cancer), one-third of non-small cell lung cancer (the most common type of lung cancer), and up to half of colorectal tumors. Colorectal, lung and pancreatic cancers are among the most costly and deadliest types of cancer to be treated. According to the World Health Organization (WHO), cancer caused 9.6 million estimated deaths in 2018. WHO also reports that in 
$2010, \$ 1.16$ trillion was estimated to be the total annual economic cost of cancer.

It is sad to know that after several decades of research, there are no inhibitors that target RAS genes (such as KRAS). It is highly problematic to obtain high quality crystals of full length KRAS proteins on Earth, and crystallization in space may produce improved crystals that can aid in structural determination, leading to the development of inhibitors to treat cancers associated with the KRAS gene. In the ISS National Lab investigation that was launched on SpaceX CRS-16, crystallization of the full length unmodified KRAS proteins, cancer causing KRAS mutants, and KRAS proteins in complex with various small molecule inhibitors were sought after ${ }^{27}$.

\section{Advancing Parkinson's research toward a cure}

Michael J. Fox Foundation partnered with the ISS National Lab in an investigation to crystallize Leucine-rich repeat kinase 2 (LRRK2) - a protein associated with the development of Parkinson's disease (a condition currently affecting 6 million people worldwide). Growing crystals of LRRK2 in microgravity, it is hoped to obtain larger crystals having fewer defects than those formed on Earth. Higher quality crystals could improve the structure determination of LRRK2, which could lead to advancements in structurebased drug design for Parkinson's. This' is the $2^{\text {nd }}$ ISS National Lab investigation from The Michael J. Fox Foundation. The $1^{\text {st }}$ investigation that launched on SpaceX CRS-12, produced crystals of high quality but were not large enough to improve LRRK2's structure determination. In the $2^{\text {nd }}$ investigation launched on Northrop Grumman CRS-10, a different type of hardware was used to allow larger crystal growth ${ }^{27}$.

\section{CONCLUSION}

This review summarizes the findings attained by groups of scientists and those of the International Space Station (ISS) on protein crystallization in microgravity. Results obtained showed that protein crystals developed in microgravity yielded better crystals compared to those obtained in the Earth's environment which were instrumental to MABs drug development as well as useful in diagnosis of some disease as they could serve as very important biomarkers used in tracing biochemical pathways.

\section{REFERENCES}

1. Walter HU, Minster O, Kufner E, Binot R, The Microgravity Applications Promotion programme of the European Space Agency, ESA. AIP Conference Proceedings 420, 1998, pg: 399. Doi:10.1063/1.54825

2. Oluwafemi F, Microgravity Research Applications in Life Sciences Using Clinostat, Lap Lambert Academic Publishing, trademark of International Book Market Service Limited, member of OmniScriptum Publishing Group, 17 Meldrum Street, Beau Bassin 71504, Mauritius, ISBN-13: 978-613-990145-6, ISBN-10: 6139901456, EAN: 9786139901456, 2018, 50pgs.
3. NASA, National Aeronautics and Space Administration, Protein Crystals in Microgravity. https://www.nasa.gov/mission pages/station/research/be nefits/mab/ [accessed 5 June 2020].

4. Somesh R, Sujata D, Prashant D, Swati S, Parag K, Rohan H, Monoclonal Antibodies in Therapeutics: Asian Journal of Biomedical and Pharmaceutical Sciences, 3, 19, 2013, 35-39.

5. Russo KI, Merlino A, Vergara A, Sica F, An Overview of Biological Macromolecule Crystallization, International Journal of Molecular Sciences, 14,6, 2013, 11643-11691. Doi: $\underline{10.3390 / i j m s 140611643}$

6. Oluwafemi FA, Space Science and Technology in Economic Prosperity, Lap Lambert Academic Publishing, trademark of International Book Market Service Limited, member of OmniScriptum Publishing Group, 17 Meldrum Street, Beau Bassin 71504, Mauritius, ISBN-13: 978-613-9-97995-0, ISBN10: 6139979951, EAN: 9786139979950, 2018, 52pgs.

7. McPherson A, and DeLucas LJ, Microgravity Protein Crystallization, npj Microgravity 1, 15010, 2015. Doi:10.1038/npjmgrav.2015.10.

8. Hoffman AS, Hydrogels for Biomedical Applications, Advanced Drug Delivery Reviews, 64, 2012, 1823. Doi:10.1016/j.addr.2012.09.010

9. Deschamps JR, The Role of Crystallography in Drug Design. The AAPS Journal 7, 4, 2005, E813-E819.

10. Braddock $M$, From Target Identification to Drug Development in Space: Using the Microgravity Assist. Current Drug Discovery Technologies, 16, 00-00, 2019. Doi: $10.2174 / 1570163816666190112150014$.

11. Blaber $E$, Marçal $H$, Burns BP, Bioastronautics: The Influence of Microgravity on Astronaut Health, Astrobiology, 10, 5, 2010, 463-473. Doi: 10.1089/ast.2009.0415

12. Jaenicke R, Hebe U, Franks F, Chapman D, Griffin MCA, Hvidt A, Cowan DA, Protein Structure and Function at Low Temperatures [and Discussion], Philosophical Transactions of the Royal Society of London, Series B, Biological Sciences, 326, 1237, 1990, 535553. Doi: $10.1098 /$ rstb. 1990.0030.

13. Laskowski RA, Protein Structure Databases. Mol Biotechnol, 48, 2, 2011, 183-198. Doi:10.1007/s12033-0109372-4.

14. Sneha P, George C, Priya D, Inflammatory Disorders Part-B, Advances in Protein Chemistry and Structural Biology $23^{\text {rd }}$ edition, 102, 1, 2016, 384 ISBN: 978-0-12-804795-8, ISSN: 1876-1623.

15. Banach M, Konieczny L, Roterman I, Secondary and Supersecondary Structure of Proteins in Light of the Structure of Hydrophobic Cores. Methods Mol. Biol. 1958, 2019, 347-378.

16. Bleiholder $\mathrm{C}$ and Liu FC, Structure Relaxation Approximation (SRA) for Elucidation of Protein Structures from Ion Mobility Measurements, Journal of Physical Chemistry, 123, 13, 2019, 2756-2769.

17. Clarke J, Berg M, John LT, Lubert S, Quaternary Structure: Polypeptide Chains Can Assemble into Multisubunit Structures, Biochemistry 5. ed., 4. print. ed., 2002, New York, Newyork: W.H. Freeman, ISBN 0-7167-3051-0. 
18. Xiao X, Wang P, Chou KC, Predicting protein Quaternary Structural Attribute by Hybridizing Functional Domain Composition and Pseudo Amino Acid Composition, Journal of Applied Crystallography, 42, 2009, 169-173.

19. McPherson A, and Gavira JA. Introduction to Protein Crystallization, Acta Crystallographica Section F Structural Biology Communications, 70,1, 2013, 2-20. Doi:10.1107/s2053230x13033141. ISSN 2053-230X.

20. Lin $Y$, What's Happened Over the Last Five Years with HighThroughput Protein Crystallization Screening? Expert Opinion on Drug Discovery, 13, 8, 2018, 691-695.

21. Smith AW, Spotlight: Microgravity Molecular Crystal Growth, ISS National Lab. https://upward.issnationallab.org/microgravity-molecularcrystal-growth/ 2017 [accessed 5 June 2020].

22. Oluwafemi F, Microgravity Research and Applications: Roles in Economic Prosperity and Poverty Reduction, Proceedings of United Nations/United Arab Emirates, High Level Forum "Space as a Driver for Socio-Economic Sustainable Development", Dubai, United Arab Emirates, 6-9 November, 2017.
23. Howard J, NASA, Space Station Crew Cultivates Crystals for Drug Development. https://phys.org/news/2017-03-spacestation-crew-cultivates-crystals.html [accessed 5 June 2020].

24. Afolayan EM, Oluwafemi FA, Jeff-Agboola EO, Oluwasegun T, Ayankale JO. Socio-Economic Benefits of Microgravity Research, Arid Zone Journal of Engineering, Technology and Environment (AZOJETE), Centre for Satellite Technology Development Special Issue: Space Science and Technology for Sustainable Development, Vol. 15(SP.i2), 2019, 57-74, Print ISSN: 1596-2490, Electron vic ISSN: 2545-5818.

25. Dunbar B, Protein Crystals in Microgravity. https://www.nasa.gov/mission pages/station/research/be nefits/mab/2017 [accessed 5 June 2020].

26. Aibara S, Shibata K, Morita $\mathrm{Y}$, Protein Crystallization in Microgravity, Biological Sciences in Space, Vol.11 No.4, 1997, 339-345.

27. Smith AW, The ISS National Lab Blog. Designing Better Drugs: Piecing Together Protein Function through Structure, https://www.issnationallab.org/blog/designing-betterdrugs-piecing-together-protein-function-throughstructure/ 2019 [accessed 5 June 2020].

Source of Support: None declared.

Conflict of Interest: None declared.

For any question relates to this article, please reach us at: editor@globalresearchonline.net New manuscripts for publication can be submitted at: submit@globalresearchonline.net and submit_ijpsrr@rediffmail.com 